\title{
Universality Classes of Chaotic Cellular Automata
}

\author{
T. G. Mattos and J. G. Moreira \\ Departamento de Física, Instituto de Ciências Exatas, Universidade Federal de Minas Gerais, \\ C. P. 702, 30123-970, Belo Horizonte, MG, Brazil
}

Received on 6 January, 2004

\begin{abstract}
Cellular automata (CA) are discrete, spatially-homogeneous, locally-interacting dynamical systems of very simple construction, but which exhibit a rich intrinsic behavior. Even starting from disordered initial configurations, CA can evolve into ordered states with complex structures crystallized in space-time patterns. In this paper we concentrate on deterministic one-dimensional CA defined by rules that lead to chaotic patterns. In order to find universality classes for these rules we associate a growth process with the CA dynamics and study the temporal behavior of the growth exponent, skewness and kurtosis of the height distribution of the interface. We obtain four universality classes characterized by different values of the growth exponent. These are related to the random deposition and directed percolation classes.
\end{abstract}

\section{Introduction}

Self-affine interfaces generated by non equilibrium surface growth have been intensively studied in the 1990's [1, 2]. One of the reasons of interest is to distinguish universality classes to which these kinetic roughening models can belong. Each universality class is related to a diffusion equation with two main terms: the noise of the process of particle deposition and the correlations of the growth process. Some classes - for example, the random deposition (RD), the directed percolation (DP) and the compact directed percolation (CDP) [3] - are characterized by the absence of correlations in the growth process. In other classes - as the KPZ [4] or EW [5, 6] classes - these correlations are fundamental.

The universality classes are characterized by the behavior of the roughness [7] - the width of the interface - and of the height distribution [8] of this interface. In the classes without correlations in the growth process, the roughness $\omega(t)$ grows with the time $t$ as a power law $-\omega \sim t^{\beta}$ - with a well defined exponent, the growth exponent $\beta$. The value of this exponent characterizes the universality class: $\beta=0.5$ in the RD class, $\beta \simeq 0.8$ in the DP class and $\beta=1.0$ in the CDP class [9].

More recently, this approach has been used to study other kinds of models such as cellular automata [9, 10, 11], sandpiles [12], and the contact process [13]. These studies are done by mapping the model into a growth process which generates an interface - the accumulation method. So, it is possible to define the height for each site of this interface and then determine the roughness, the growth exponent and the skewness and kurtosis of the height distribution.

In this work, we use this technique to study a group of cellular automata which have a chaotic behavior, that is, their evolution has a strong dependence on the initial conditions. A cellular automaton (CA) is a dynamical system that is discrete in space, in time and in the number of states. It provides a simple model for a great number of problems in science - chemical reactions, crystal growth models, turbulence, neural networks, biological systems, or other nonlinear process far from equilibrium [14]. In this automaton, each site (noted $i$ ) is associated to a variable $\sigma_{i}$, which can be in $k$ different states $\sigma_{i}=0,1, \ldots, k-1$. The dynamics is defined, at each time step, by rules depending on the values of $\left\{\sigma_{i}\right\}$ at previous time, associated to a given number of $r$ arbitrary neighbor sites. The local rules of a CA may be either probabilistic or deterministic and the states of the sites are simultaneously updated.

Based on the spatiotemporal patterns generated by CA evolution, Wolfram [14] developed a classification scheme consisting of four qualitative classes: homogeneous fixed point (class I), periodic (class II), chaotic (class III), and complex (class IV). In this paper, CA in Wolfram's class III are investigated using the accumulation method. Our goal is to distinguish different universality classes in the chaotic class of these automata. Section 2 introduces the definitions of the automaton, the accumulation method and the statistic relevant quantities. In section 3, we show that there are four distinct universality classes in the chaotic Wolfram's class. Finally, we draw some conclusions in section 4.

\section{Definitions}

Wolfram's CA consists of a one-dimensional chain of $L$ lattice sites $(i=1,2, \ldots, L)$, with periodic boundary conditions. We study the automaton with $k=2$, that is, each site has two possible states $\sigma_{i}=0,1$ and the state of the system at time $t$ is specified by the set $\left\{\sigma_{i}(t)\right\}$. At the next time step the state of a given site is $\sigma_{i}(t+1)$, depending on the states of the first neighbors $(r=1), \sigma_{i-1}(t), \sigma_{i}(t)$ and $\sigma_{i+1}(t)$. The states of all sites are simultaneously updated at each time step - synchronous update. So, for this local neighborhood of three sites there are $2^{3}=8$ possible configurations, and with each one of them a CA rule can associate either 
0 or 1 with state $\sigma_{i}$ at time $t+1$. Thus, one can construct $2^{8}=256$ deterministic local rules for CA with a binary state variable and a neighborhood of three sites. All the 256 Boolean functions that specifies the deterministic CA rules are labelled by a decimal code [14] obtained in the following way: we write the eight outcomes associated with the local neighborhoods ordered in decreasing order, and read them as the eight binary digits of the CA rule code. Rule 90, for example, is defined by the configuration given in Fig. 1.

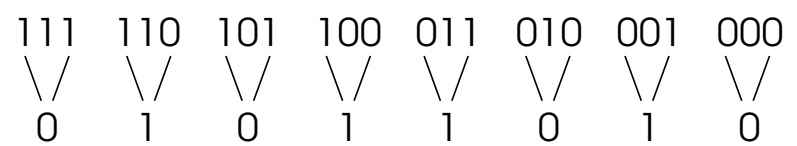

Figure 1. The eight possible configurations for a neighborhood of three sites at time $t$ and the state associated to each one by CA rule 90 , at time $t+1$. Note that the sequence 01011010 is the binary representation of the decimal 90.

Based on the spatiotemporal patterns generated by CA evolution, Wolfram developed a classification scheme consisting of four qualitative classes: homogeneous fixed points (class I), periodic (class II), chaotic (class III) and complex (IV) [14]. Rules in Wolfram's class III are characterized by a strong dependence on the initial conditions: if one makes a copy of the system introducing some alterations (damage) and lets both systems evolve following the same deterministic rule, one can identify chaotic behavior by monitoring the Hamming distance, defined as

$$
D_{H}(t)=\frac{1}{L} \sum_{i=1}^{L}\left|\sigma_{i}(t)-\sigma_{i}^{\prime}(t)\right|
$$

where the set $\left\{\sigma_{i}^{\prime}\right\}$ is the damaged copy of $\left\{\sigma_{i}\right\}$ at $t=0$. By definition [16], chaotic systems have nonzero stationary Hamming distance $D_{H}(\infty)$.

We are interested in the study of all rules [15] in Wolfram's class III so, we associate a growth process to the time evolution of the CA rules and use the techniques developed in the study of such processes. In the accumulation method the height $h_{i}(t)$ associated with site $i$ at time $t$ is defined as

$$
h_{i}(t)=\sum_{\tau=1}^{t} \sigma_{i}(\tau) .
$$

In particular we study the height distribution around the average height, for which we can define the n-th moment at time $t$ as

$$
\mu_{n}(t)=\frac{1}{L} \sum_{i=1}^{L}\left[h_{i}(t)-\bar{h}(t)\right]^{n}
$$

where

$$
\bar{h}(t)=\frac{1}{L} \sum_{i=1}^{L} h_{i}(t)
$$

is the average height of the distribution at time $t$. The second moment is a measure of the width of the interface and we define the roughness at time $t$ as its square root,

$$
\omega(t)=\mu_{2}^{\frac{1}{2}}(t)
$$

Generally, in growth processes, the roughness grows as a power law,

$$
\omega \sim t^{\beta}
$$

where $\beta$ is the growth exponent.

Other relevant quantities to characterize the distribution [8] are the skewness, defined as

$$
S(t)=\frac{\mu_{3}(t)}{\mu_{2}^{\frac{3}{2}}(t)},
$$

which is a measure of the asymmetry of the distribution, and the kurtosis,

$$
K(t)=\frac{\mu_{4}(t)}{\mu_{2}^{2}(t)}-3
$$

which is a measure of the flatness of the distribution. For a Gaussian distribution we have $S=K=0$.

A universality class of a growth process is defined by the associated symmetry properties and the conservation laws. The growth equation that describes the system can present scaling laws, power laws or some symmetry, which lead to a quantitative characterization of the universality class.

As an example, we consider the random deposition (RD) model [1]. Particles are deposited randomly at a constant rate on a $d$-dimensional discrete substrate ( $L^{d}$ lattice sites). This is a very simple model, as we see that the process is uncorrelated because the height associated to a given site grows independently from the others. The growth equation for this process is

$$
\frac{\partial h(\vec{x}, t)}{\partial t}=F+\eta(\vec{x}, t)
$$

where $F$ is the average number of particles deposited on site $\vec{x}$ (deposition rate per site) and $\eta(\vec{x}, t)$ is a white noise (zero average, $\delta$-correlated in space and time) that represents the randomness of the deposition process. The solution for this equation is exact and leads to a power law for the temporal behavior of the roughness

$$
\omega \sim t^{\beta}
$$

with $\beta=1 / 2$. For the symmetry of the deposition process, explicitly given by the white noise in the growth equation, the skewness and kurtosis of the height distribution are both zero. Thus, the growth exponent $\beta=1 / 2$, together with $S=K=0$, defines a universality class: the random deposition class. 


\section{Simulations Results}

We made simulations of systems with $L=10^{4}$ with periodic boundary conditions, until $10^{5}$ time steps, averaging over 100 samples. For some rules we made a finite size analysis to find the values of the growth exponent with better precision. For some other rules, we also made an asymptotic study with $L=10^{3}$ until $10^{7}$ time steps. The initial condition used in the simulations is random, with half the sites occupied. We considered all 38 rules in Wolfram's class III [14].

We used the growth exponent, skewness and kurtosis to find universality classes and, considering the growth exponent, four universality classes were found, as one can see in Fig. 2.

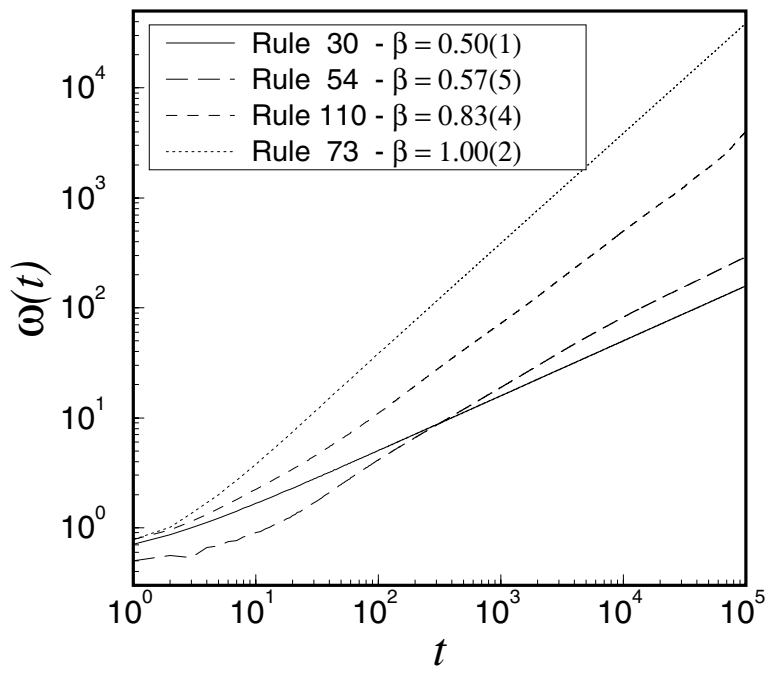

Figure 2. Log-log plot of roughness $\omega(t)$ versus time $t$, where the slope is the growth exponent $\beta$. We identify four distinct values for rules in Wolfram's class III, each one corresponding to a different universality class.

In the first class, corresponding to the CDP universality class, we have rules 73 and 109 , with $\beta=1.00 \pm 0.02$. This value of the growth exponent, meaning that the roughness grows linearly with time, can be understood as follows: clusters of $0 s$ and $1 s$ are formed and the heights associated with the clusters of $1 s$ grows one unit in each time step, while the heights associated with the clusters of $0 s$ do not grow. So, the interface width grows one unit at each time step and the height distribution is obviously not symmetric, thus we found non-zero values of the skewness and kurtosis.

In the second class, we have rules $110,124,137$ and 193 , with $\beta=0.83 \pm 0.01, S=0$ and $K<0$. This value for the growth exponent, close to the value of DP universality class, is also obtained in the frozen-active transition in the probabilistic Domany-Kinzel cellular automaton (DKCA) [9] when a symmetric updating scheme is applied. For these rules, we found $S=0$ for $t>10^{4}$ and $K<0$ for all $t$.

Rules 54 and 147 define the third class, with $\beta=$ $0.66 \pm 0.01$. As one can see in Fig. 2, the power-law behavior only occurs after approximately $10^{2}$ time steps. This value of the growth exponent is close to the exponent obtained in the frozen-active transition in the DKCA when the non-symmetric updating scheme is applied [9]. Here we have $S \neq 0$ and $K>0$. In the asymptotic study we note that a size independent crossover occurs for $10^{4}<t<10^{5}$ and an exponent $\beta=0.53 \pm 0.01$ is obtained. When this regime is reached, we have $S=K=0$. Both second and third classes correspond to systems described by a growth equation with non-white noise.

For the other 30 rules, we found $\beta=0.50 \pm 0.01$. We could distinguish four subclasses for these rules, by analysis of the temporal behavior of the skewness and kurtosis, as one can see in Fig. 3. In the first subclass we have rules 30, $45,60,75,86,89,90,101,102,105,106,120,135,149$, $150,153,165,169,195$ and 225, for which we found $S=0$ at all times and $K \rightarrow 0$ very quickly $\left(t \sim 10^{2}\right)$. These rules are in the RD universality class for all times, and the growth equation describing the evolution of these systems has white noise. The other rules present a different behavior of skewness and kurtosis in the beginning of the process, but go asymptotically to the RD universality class. We can separate them in three distinct subclasses, according to this initial behavior. For rules 18 and 183, kurtosis and skewness oscillate as they go to zero, reaching this value after about $10^{2}$ time steps for the skewness and after $10^{3}$ time steps for kurtosis. These quantities for rules 22, 146, 151 and 182 do not oscillate, but take a longer time to go to zero. Rules 122 , 126, 129 and 161 quickly reach null skewness and kurtosis, after about $10^{2}$ time steps.

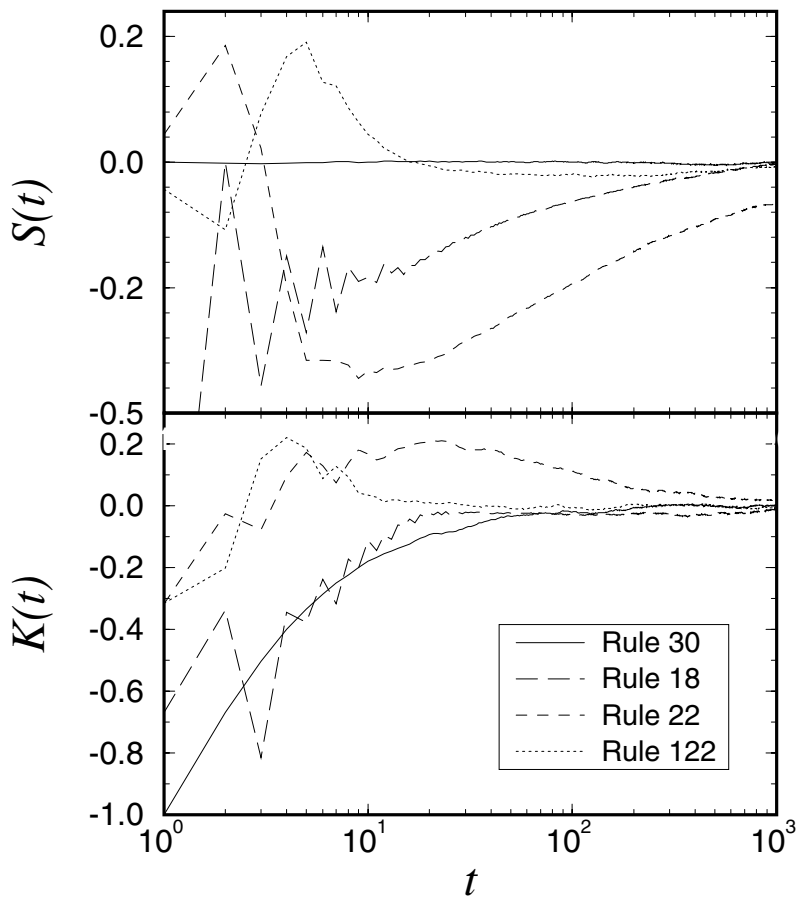

Figure 3. Semi-log plots of skewness $S(t)$ (top), and kurtosis $K(t)$ (bottom), as functions of time $t$, for rules with $\beta=0.5$. We see four different behaviors in the beginning of the growth process. 


\section{Conclusions}

We divide chaotic rules in Wolfram's class III in four different universality classes, through the association of a growth process to the dynamics of the CA rules and determination of the growth exponents. Many of these rules are in the RD class, some of them asymptotically. Other rules are in the CDP class, in which one can identify clusters of $0 s$ and $1 s$. The remaining rules appear to be in the same class as the frozen-active transition of the DKCA, some in the symmetric updating scheme and other in the non-symmetric scheme.

\section{Acknowledgements}

We thank R. Dickman for helpful criticism of the manuscript. This work is supported by Brazilian agencies $\mathrm{CNPq}$ and FAPEMIG.

\section{References}

[1] A.-L. Barabási and H. E. Stanley, Fractal Concepts in Surface Growth, Cambridge Univ. Press, Cambridge (1995)

[2] P. Meakin, Fractals, Scaling and Growth Far from Equilibrium, Cambridge Univ. Press, Cambridge (1998).

[3] P. Grassberg, J. Stat. Phys. 79, 13 (1995).

[4] M. Kardar, G. Parisi, and Y.-C. Zhang, Phys. Rev. Lett. 56, 889 (1986).

[5] S. F. Edwards and D. R. Wilkinson, Proc. R. Soc. A 381, 17 (1982).
[6] F. Family, J. Phys. A 19, L441 (1986).

[7] F. Family and T. Vicsek, J. Phys. A 18, L75 (1985).

[8] J. Krug, P. Meakin, and T. Halpin-Healey, Phys. Rev. A 45, 638 (1992).

[9] A. P. F. Atman, R. Dickman, and J. G. Moreira, Phys. Rev. E 66, 016113 (2002);

[10] J. A. de Sales, M. L. Martins, and J. G. Moreira, J. Phys. A 32, 885 (1999).

[11] A. P. F. Atman and J. G. Moreira, Eur. Phys. J. B 16, 501 (2000); Phys. Rev. E 67, 016107 (2003).

[12] A. Vespigniani, R. Dickman, M. A. Muñoz, and S. Zapperi, Phys. Rev. E 62, 4564 (2000); R. Dickman, M. Alava, M. A. Muñoz, J. Peltola, A. Vespigniani and S. Zapperi ibid 64, 056104 (2001)

[13] R. Dickman, M. A. Muñoz, Phys. Rev. E 62, 7632 (2000).

[14] S. Wolfram, Rev. Mod. Phys. 55, 601 (1983); S. Wolfram, Theory and Applications of Cellular Automata (World Scientific, Singapore, 1986).

[15] Wolfram only studied the "legal" rules, that is, the rules which do not have spontaneous generation (if, at time $t$ the local neighborhood is 000 , the state at time $t+1$ is 0 ) and they have simmetry (the configurations 110 and 011 or the configurations 100 and 001 have the same future).

[16] C. Tsallis, in: L. Blum (Ed.), Condensed Matter Theories, Plenum, New York, 1992. 